\title{
Magnetic resonance angiography without contrast enhancement medium in bone and soft tissue tumors
}

\author{
HIDEYUKI DOI $^{1}$, ATSUSHI ONO ${ }^{2}$, AKIRA KAWAI ${ }^{3}$, YUKI MORIMOTO ${ }^{3}$, \\ TOSHIYUKI KUNISADA ${ }^{1}$, EIJI NAKATA ${ }^{1}$ and TOSHIFUMI OZAKI ${ }^{1}$ \\ ${ }^{1}$ Science of Functional Recovery and Reconstruction, Okayama University Graduate School of Medicine, Dentistry, and \\ Pharmaceutical Sciences, Okayama 700-8558; ${ }^{2}$ Department of Medical Technology, Okayama Kosei-Hospital, Okayama; \\ ${ }^{3}$ Department of Orthopaedic Surgery, National Cancer Center Hospital, Tsukiji, Chuo-ku, Tokyo 104-0045, Japan
}

Received August 19, 2005; Accepted November 25, 2005

\begin{abstract}
The aim of this study was to assess the ability of a new magnetic resonance (MR) angiography technique that does not use contrast enhancement medium to depict the vascularity of musculoskeletal neoplasms, and evaluate its clinical utility. We performed 3D fresh blood imaging (FBI) MR angiography in 57 patients with bone or soft tissue tumors, and the detection of vessels in and around the tumor was evaluated. Moreover, differences in vascularity between benign and malignant tumors were analyzed. In the lower leg, large arteries such as femoral or popliteal arteries were visible. In the trunk or arm, large vessels such as subclavian or iliac arteries were visible. Discrimination between benign and malignant tumors was impossible in bone tumors; however, the mean value of vascularity differed between benign and malignant tumors in the soft tissue tumors. This is the first trial of the FBI method for bone and soft tissue tumors. The still developing method of MRA without contrast materials could clearly depict major arteries in the trunk and the extremities, this method may replace conventional MRA of bone and soft tissue tumors because it produces vivid images while being non-invasive.
\end{abstract}

\section{Introduction}

The introduction of intensive chemotherapy has improved the prognosis of patients with bone and soft tissue sarcoma (1-4). Limb-sparing surgery has become possible in most cases of sarcomas involving the extremity. In planning limb

Correspondence to: Dr Toshifumi Ozaki, Science of Functional Recovery and reconstruction, Okayama University Graduate School of Medicine, Dentistry, and Pharmaceutical Sciences, Okayama 700-8558, Japan

E-mail: tozaki@md.okayama-u.ac.jp

Key words: magnetic resonance imaging, fresh blood imaging, angiography, contrast enhancement medium, bone and soft tissue tumors preservation surgery, precise evaluation of tumor extent and the relationship between the tumor and vessels or nerves is important. Moreover, estimation of local blood flow is also important for surgical planning along with evaluating the response to preoperative chemotherapy (5). Angiography is an attractive method of preoperative planning for sarcoma excision. If angiography could be done without invasion, similar to unenhanced magnetic resonance (MR) imaging, it would be the optimal method for preoperative planning.

There are 4 types of MR angiography methods. The time of flight (TOF) method is commonly used for the evaluation of intra- and extracranial circulations. Better results in the identification of distal runoff vessels have been reported with the 2D TOF technique than with conventional arteriography (6). However, this method is not suited to imaging arteries in a large field of view due to the long acquisition time and saturation effects $(7,8)$. The phase contrast (PC) method can image vessels in a large anatomic area, such as the lower extremity; however, PC also requires a long acquisition time, and turbulent flow and venous overlay affect the quality of images in a distal region $(8,9)$. As the contrast-enhanced (CE) method is easy and images are obtained quickly, it has gained widespread clinical acceptance for evaluating the aorta and major arteries. Although this image provides useful vascular information, the economic cost of the contrast material is high $(10,11)$. In addition, anaphylactic reactions are always possible even though the risk is much lower for paramagnetic contrast agents than for the iodinated contrast used for conventional angiography (7).

We studied a modification of a new MR angiography technique without contrast enhancement for bone and soft tissue tumors. This technique is called fresh blood imaging (FBI), which uses 3D half-Fourier fast spin-echo (FSE) triggered by electrocardiograph (ECG) gating. This technique allows good delineation of arteries. Several authors have described its clinical usefulness in patients with aortic and arterial diseases $(11,12)$. However, there are no clinical trials of the FBI method for musculoskeletal neoplasms. This study assessed the quality of visualization of local vessels in and around the tumor by unenhanced MR angiography (MRA). Moreover, the discrimination between benign and malignant tumors was attempted. 


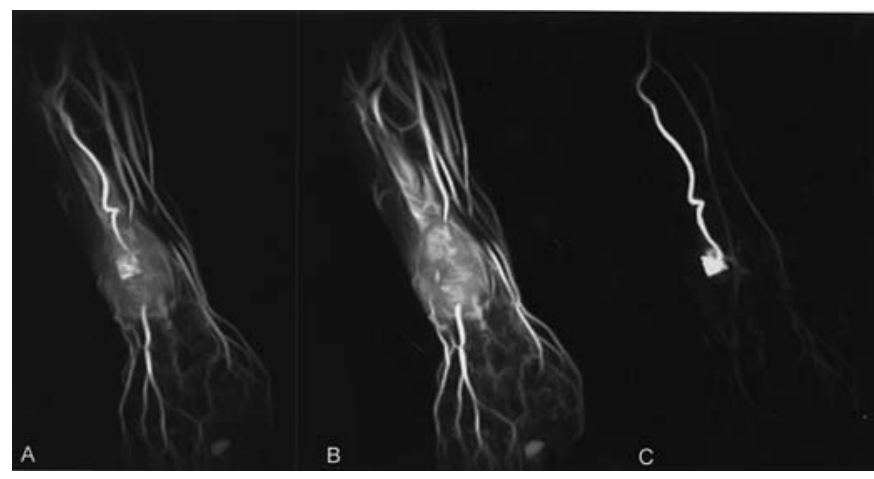

Figure 1. A 51-year-old female with hemangioma of the forearm. The first MR image obtained during the diastolic phase showed arteries and veins with a slow flow (A). The second MR image obtained during the systolic phase showed veins (B). Finally, the MR image obtained after diastolic and systolic subtraction depicted only arteries (C).

TableI. Visible arteries on MRA.

\begin{tabular}{lcr}
\hline Location & Visible rate & $\%$ \\
\hline Upper extremity & & \\
Brachial artery & $3 / 7$ & $43 \%$ \\
Ulnar artery & $3 / 7$ & $43 \%$ \\
Radial artery & $3 / 7$ & $43 \%$ \\
$\quad$ Interosseus artery & $2 / 7$ & $29 \%$ \\
Trunk & & \\
$\quad$ Subclavian artery & $7 / 7$ & $100 \%$ \\
Iliac artery & $16 / 16$ & $100 \%$ \\
Lower extremity & & \\
Femoral artery & $21 / 21$ & $100 \%$ \\
Profunda femoris artery & $18 / 18$ & $100 \%$ \\
Lat. circum. femoral artery & $15 / 18$ & $83 \%$ \\
Genicular artery & $18 / 21$ & $86 \%$ \\
Popliteal artery & $21 / 21$ & $100 \%$ \\
Ant. tibial artery & $19 / 23$ & $83 \%$ \\
Post. tibial artery & $19 / 23$ & $83 \%$ \\
$\quad$ Peroneal artery & $13 / 16$ & $81 \%$ \\
\hline
\end{tabular}

\section{Materials and methods}

Subjects. There were 31 bone tumors and 26 soft tissue tumors analyzed with this method between August 2001 and February 2003. The 31 bone tumors included 14 osteosarcomas, 4 enchondromas, 2 metastatic cancers, 2 chondrosarcomas, 2 giant cell tumors, 2 osteochondromas, 2 non-ossifying fibromas, 1 adamantinoma, 1 chondromyxoid fibroma and 1 solitary bone cyst. The 26 soft tissue tumors included 5 liposarcomas, 4 schwannomas, 4 synovial sarcomas, 3 MFHs, 2 metastatic cancers, 2 lipomas, 2 PVNS, 1 hemangioma, 1 myxoma, 1 ganglion, and 1 extra-abdominal desmoid. Of the tumors, 12 were located in the upper extremities, 7 in the trunk, and 38 in the lower extremities. There were 32 males and 25 females ranging in age between 10 and 75 years (average, 39.8 years). Informed consent was obtained from all patients prior to inclusion in the study.

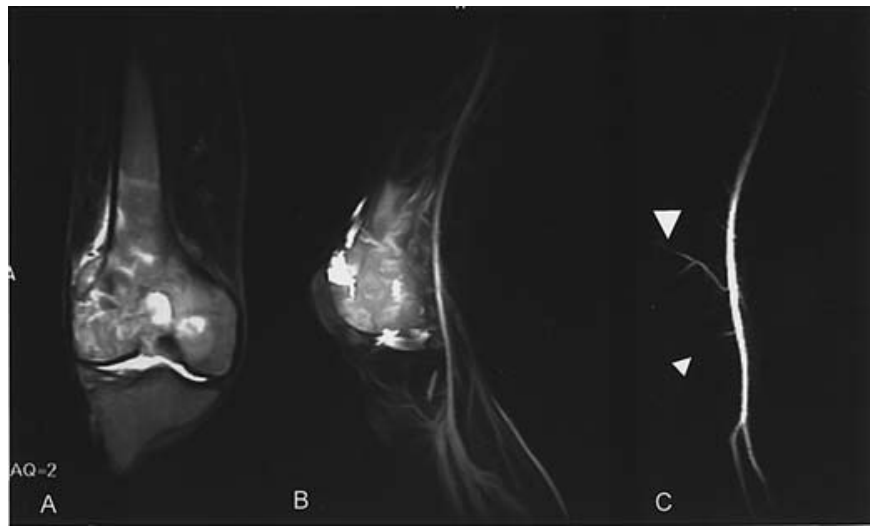

Figure 2. A 21-year-old male with osteosarcoma of the distal femur. (A) T2weighted image. (B) Artery and vein images of MR angiography. (C) Artery image of MR angiography. Tumor hypervascularity (small arrow) and the involvement of arteries (large arrow) were detected.

MR imaging. The FBI experiments were performed using a 0.5-T clinical MR imager (FlexArt/Hyper, Toshiba, Japan). A whole-body QD coil was used, and the coronal in-plane acquisition was obtained using TR $=3 \mathrm{R}-\mathrm{R}$, TEeff $=39 \mathrm{msec}$, $\mathrm{ETS}=6.5 \mathrm{msec}, \mathrm{TI}=140 \mathrm{msec}, 256 \times 256$ matrix, 2 shots, 15-30 partitions with a 3.5-thick slice, $\mathrm{NAQ}=1$, and FOV $=$ $36-42 \times 36-42 \mathrm{~cm}$. The FBI technique allows the delineation of slow flow vessels during the cardiac phase; hence, arteriovenous images were acquired with diastolic phase images and venous images were acquired with systolic phase images, respectively. Prior to 3D acquisition, a single slice at multiple phases (ECG-Prep scan) was obtained to determine appropriate diastolic and systolic phases. After both systolic and diastolic 3D images were acquired, subtraction of systole from diastole gives arterial images followed by an MIP procession. Fig. 1 shows typical FBI images acquired with patient. The FBI experiments were performed before diagnostic biopsy or preoperative treatment. The simultaneous MRI protocol included T1- and T2-weighted spin echo images, and finally dynamic gadolinium-enhanced T1weighted images.

Image analysis. The ability of this modality to detect major arteries in and around the tumors was assessed. Three experienced orthopedic oncologists evaluated the images.

In order to discriminate between benign and malignant tumors, the evaluation of vascularity was performed with Image ${ }^{\circledR} \mathrm{v} 1.29$, an image analysis program (National Institute of Health, Bethesda, MD) in the tumor area (13). After the targeted field was defined in the arterio-venous phase of MRA, the intensity of the field in the artery phase was calculated by ImageJ. The obtained intensity of the artery phase was divided by that of air outside the patient. These results were defined as the signal-to-noise ratio (SNR). We evaluated SNR comparing malignant tumors and benign tumors in bone tumors and soft tissue tumors, respectively. We excluded hemangiomas because these tumors are easy to identify due to their extremely high SNR.

The significance of differences in the ratio between or among groups was evaluated by Mann-Whitney U test. P-values $<0.05$ were considered significant. 


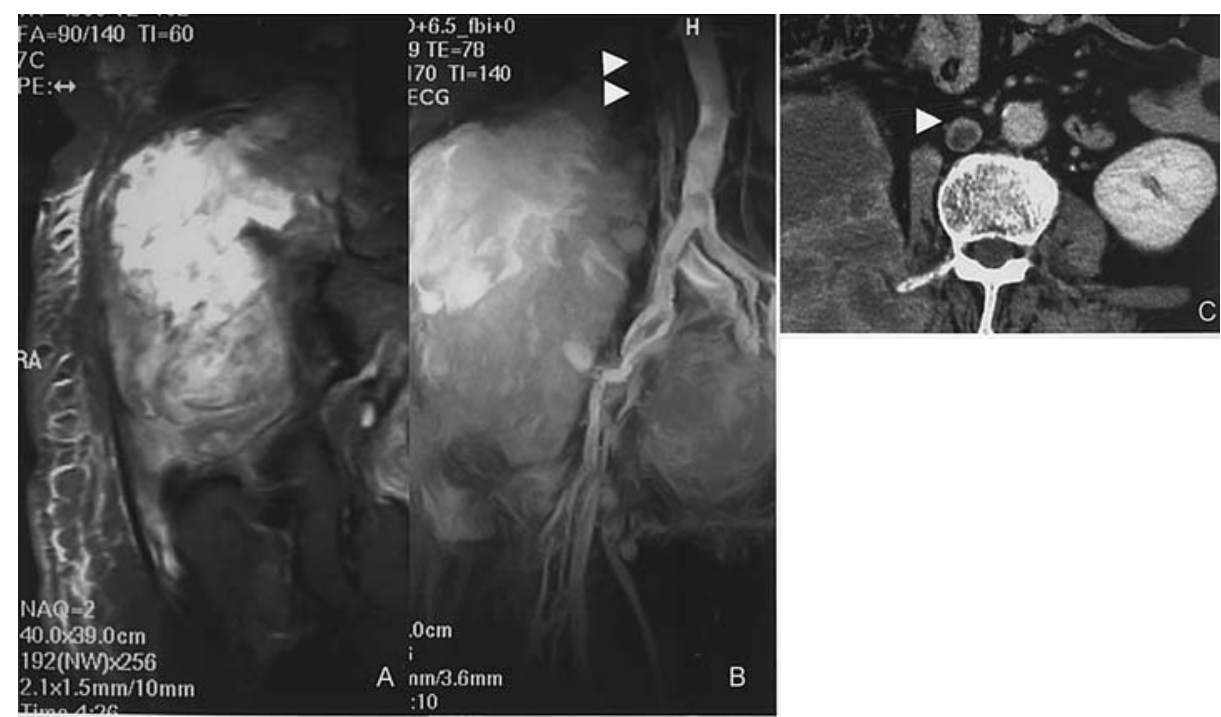

Figure 3. A 64-year-old female with large pelvic osteosarcoma. (A) T2-weighted image. (B) Artery and vein images of MR angiography; arrows indicate venous thrombus in the inferior vena cava (arrow heads). (C) Enhanced CT scanning; arrow indicates venous thrombus in the inferior vena cava (arrow heads).

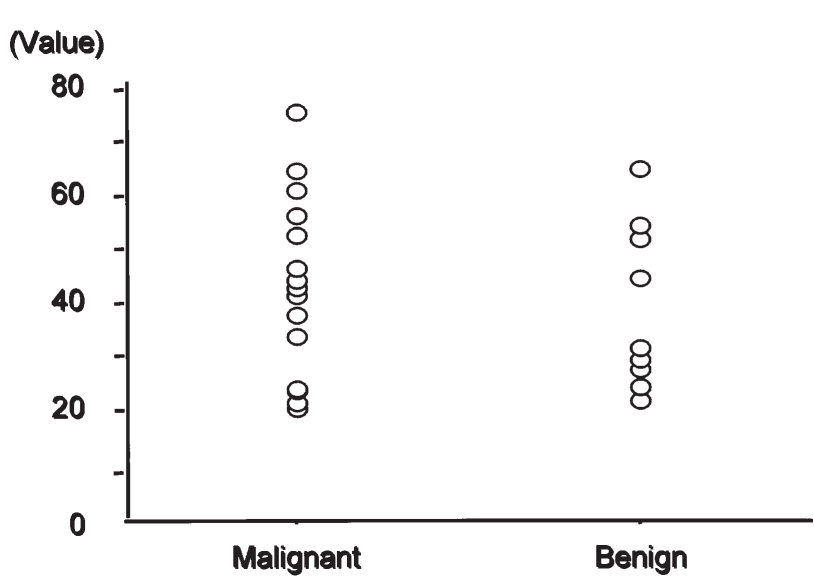

Figure 4. Vascularity of bone tumors. In bone tumors, the median value of vascularity was 48 in malignant tumors and 42 in benign tumors. Discrimination between benign and malignant tumors was difficult in bone tumors from the point of vascular conditions.

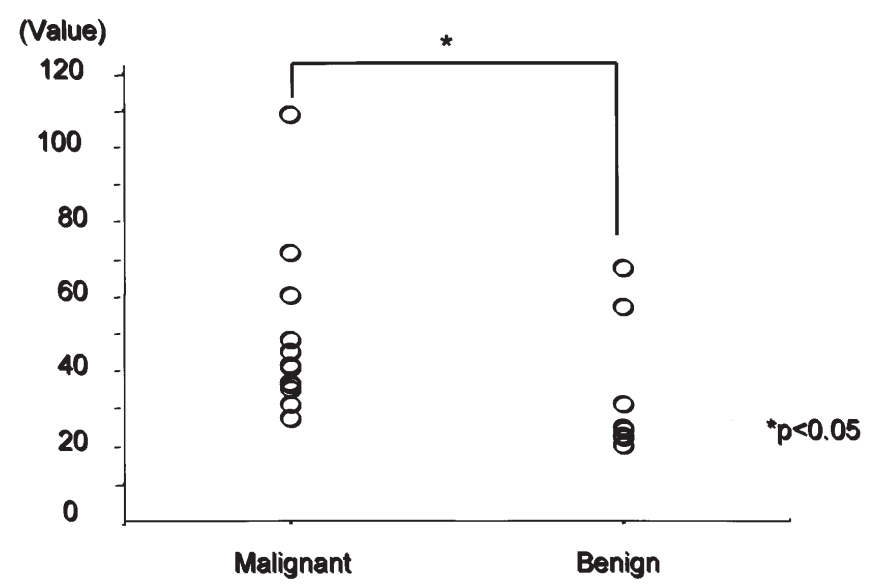

Figure 5. Vascularity of soft tissue tumors. In soft tissue tumors, the median value of vascularity was significantly different between malignant and soft benign tumors. Hemangioma was excluded from the benign tumors.

\section{Results}

The findings of each artery around the tumor were checked on FBI images. The rate of vessel identification is shown in Table I. Vascular diameter appears to influence visualization at this point in the development of the technique. In the leg, large arteries were depicted. In the trunk or arm, large vessels such as the subclavian or iliac arteries were observed; other vessels were not sufficiently visible due to their small diameter and possibly the movement of the patient.

MRA of hemangioma of the forearm shows clear tumor stain and feeding artery (Fig. 1C). Fig. 2 shows one example of hypervascularity around the tumor and involvement of the arteries. Fig. 3 also shows involvement of vessels in large pelvic osteosarcoma. In this case, tumor venous thrombus was detected in the inferior vena cava from the arterialvenous phase MRA, afterwards CT scan can also detect the thrombus.

From the intensity of vascular conditions evaluated by ImageJ, discrimination between benign and malignant bone tumors was difficult (Fig. 4). However, the SNR for soft tissue tumors excluding hemangioma significantly differed between malignant and benign soft tissue tumors $(\mathrm{p}=0.007)$ (Fig. 5).

\section{Discussion}

As flow imaging techniques, TOF and PC MR angiography may be useful, however, the acquisition time becomes quite long and flow-related artifacts influence the image quality when the region of interest is large. In the CE MRA technique, which is rapidly gaining clinical importance due to its high signal-to-noise ratio (SNR) and contrast-to-noise ratio (CNR), the contrast medium is indispensable. Furthermore, this technique critically depends on the precise bolus timing to synchronize acquisition of central k-space data with the peak of arterial enhancement (14). Inaccurate bolus timing causes ringing artifacts, reduction of SNR and venous enhancement $(15,16)$. 
The non-contrast-enhanced MRA technique using 3D half-Fourier fast spin-echo (FSE) triggered with electrocardiograph (ECG) gating was developed by Miyazaki et al $(9,17)$. Fresh blood imaging (FBI), one of the newer and most important MRA techniques, has the advantage of not requiring contrast medium and being non-invasive, and the required number of slices can be reduced by imaging along the coronal plane, thus shortening the acquisition time (18).

Angiography is usually performed to investigate tumor vascularity and the relationship between vessels and tumors. The usefulness of angiography in planning surgical treatment of bone tumors has been reported previously (19). Fujii et al also reported that digital subtraction angiography (DSA) is a sensitive method of estimating the histologic effect of chemotherapy (5). DSA can show tumor vascularity clearly, but this method is associated with intervention, irradiation, and numerous complications such as thrombosis. As the authors performed DSA in more than 200 patients, it was fortunate that we did not encounter venous thrombosis in the present study. In the 1990s, Swan et al (20) and Lang et al (21) reported the usefulness of MR angiography with contrast medium for surgical planning for bone and soft tissue tumors. The value of MR angiography for monitoring the response to chemotherapy was also reported by Lang et al (21).

In the current study, the imaging of major arteries in the lower extremities or trunk was superior to that of the upper extremities. In the peripheral vessels of some cases, the arterial pulsation was weakened with slow blood flow, the difference of flow speed was reduced between the systolic and diastolic phase, and the separation of arteries from veins became worse (22). Thus, arteries were imaged during the systolic phase, increasing the difficulty in diagnosing venous disease (18). However, in one case of pelvic osteosarcoma, tumor thrombus was clearly detected in the vena cava. MR venography by the FBI method can provide useful information in a short period of time in patients with diseased veins in the iliac region and lower extremity (18). This method could be widely applied to the non-invasive detection of venous thrombosis.

Discrimination of benign and malignant bone tumors was not clear using MRA, probably because benign bone tumors include tumors with rich vascularity, such as giant cell tumors. MRA may therefore be more useful for the differentiation of malignant and benign soft tissue tumor. These analyses must await further development of this imaging technique.

The MRA used in the current study has several advantages. Compared to DSA or CTA or contrast-enhanced MRA, it is non-invasive; unlike DSA and CTA, this technique does not involve radiation exposure. T2-weighted fast spin-echo image and MRA data are collected simultaneously, and the technique can image tumors under a metal implant and map the relationship between the tumor and vessels. However, the cost of contrast materials is still problematic with an MR examination and a 20-ml bottle of contrast material costing approximately $\$ 150.00$ and $\$ 170.00$, respectively, in Japan (11).

This method provides optimal analysis from a medicaleconomic perspective. Although the CE MRA technique cannot be conducted simultaneously when dynamic MRI study is needed for diagnosis of the tumor since contrast medium is used in the former, it would be possible to perform the FBI method simultaneously. Moreover, the acquisition time is short (2-5 $\mathrm{min}$ ), and useful information about the arteries in and around the tumors can thus be obtained quickly.

However, this technique is not suitable for patients with arrhythmia because ECG triggering is used (11). Problems associated with the 3D-FBI method include elevation of the background signal in cases of severe edema, and the presence of joint effusion and fluid in the gastrointestinal tract appear to obstruct diagnosis (18).

In the future, MRA is expected to improve imaging techniques, application to the assessment of response to chemotherapy, better discrimination of arteries and veins, and cost effectiveness. We applied a new MR angiography technique without contrast medium to bone and soft tissue tumors. MRA without contrast materials could depict major arteries of bone and soft tissue tumors in the trunk and extremities. Further improvement of the technique is necessary for application to bone and soft tissue tumors in the peripheral extremities.

\section{Acknowledgements}

The authors would like to thank the medical staff at the Department of Medical Technology in Kosei Hospital for their technical assistance. This work was supported in part by a Grant-in-Aid for Cancer Research from the Ministry of Health, Labor, and Welfare (12-11), a Grant-in-Aid for General Scientific Research from the Ministry of Education, Science, Sports, and Culture (no. 14370464), and a Grant-in-Aid from the Japan Orthopaedics and Traumatology Foundation Inc. (J.O.T.F. grant no. 0134).

\section{References}

1. Winkler K, Bielack S, Delling G, Salzer-Kuntschik M, Kotz R, Greenshaw C, Jurgens H, Ritter J, Kusnierz-Glaz C, Erttmann R, et al: Effect of intraarterial versus intravenous cisplatin in addition to systemic doxorubicin, high-dose methotrexate, and ifosfamide on histologic tumor response in osteosarcoma (study COSS-86). Cancer 66: 1703-1710, 1990.

2. Rosen G: Neoadjuvant chemotherapy for osteogenic sarcoma: a model for the treatment of other highly malignant neoplasms. Recent Results Cancer Res 103: 148-157, 1986.

3. Rosen G: An opinion supporting the role of high-dose methotrexate in the treatment of osteosarcoma. Cancer Treat Res 62: 49-54, 1993.

4. Bacci G, Ferrari S, Longhi A, Forni C, Bertoni F, Fabbri N, Zavatta $\mathrm{M}$ and Versari M: Neoadjuvant chemotherapy for high grade osteosarcoma of the extremities: long-term results for patients treated according to the Rizzoli IOR/OS-3b protocol. J Chemother 13: 93-99, 2001.

5. Fujii J, Ozaki T, Kawai A, Kunisada T, Sugihara S and Inoue H: Angiography for assessment of preoperative chemotherapy in musculoskeletal sarcomas. Clin Orthop 360: 197-206, 1999.

6. Owen RS CJ, Baum RA, Perloff LJ and Cope C: Magnetic resonance imaging of angiographycally occult runoff vessels in peripheral arterial occlusive disease. N Engl J Med 326: 1577-1581, 1992.

7. Ruehm SG, Goyen M, Barkhausen J, Kroger K, Bosk S, Ladd ME and Debatin JF: Rapid magnetic resonance angiography for detection of atherosclerosis. Lancet 357: 1086-1091, 2001.

8. Steffens JC, Link J, Muller-Hulsbeck S, Freund M, Brinkmann G and Heller M: Cardiac-gated two-dimensional phase-contrast MR angiography of lower extremity occlusive disease. Am J Roentgenol 169: 749-754, 1997. 
9. Miyazaki M, Sugiura S, Tateishi F, Wada H, Kassai Y and Abe H: Non-contrast-enhanced MR angiography using 3D ECGsynchronized half-Fourier fast spin echo. J Magn Reson Imaging 12: 776-783, 2000.

10. Kita M, Mitani Y, Tanihata H, Kita K, Sato M, Takizawa O and Laub G: Moving-table reduced-dose gadolinium-enhanced three-dimensional magnetic resonance angiography: velocitydependent method with three-phase gadolinium infusion. J Magn Reson Imaging 14: 319-328, 2001.

11. Urata J, Miyazaki M, Wada H, Nakaura T, Yamashita Y and Takahashi M: Clinical evaluation of aortic diseases using nonenhanced MRA with ECG-triggered 3D half-Fourier FSE. J Magn Reson Imaging 14: 113-119, 2001.

12. Ono A, Miyazaki M, Takata S, Nakai M, Tateishi A, Kuriyama M, Kawakami Y, Taniguchi T, Shibutani O, Watanabe N, et al: Evaluation of arterial occlusive diseases in lower extremity using non-contrast-enhanced MR arteriography with ECG triggered 3D half-Fourier FSE: initial experience at $0.5 \mathrm{~T}$ : The 11th Meeting of the International Society for Magnetic Resonance in Medicine, Toronto, Abst, p1713, 2003.

13. Sherwood RJ, Rowley RB and Ward SC: Relative placement of the mandibular fossa in great apes and humans. J Hum Evol 43: 57-66, 2002.

14. Prince MR: Gadolinium-enhanced MR aortography. Radiology 191: 155-164, 1994.

15. Prince MR, Chabra SG, Watts R, Chen CZ, Winchester PA, Khilnani NM, Trost D, Bush HA, Kent KC and Wang Y: Contrast material travel times in patients undergoing peripheral MR angiography. Radiology 224: 55-61, 2002.
16. Maki JH, Prince MR, Londy FJ and Chenevert TL: The effects of time varying intravascular signal intensity and $\mathrm{k}$-space acquisition order on three-dimensional MR angiography image quality. J Magn Reson Imaging 6: 642-651, 1996.

17. Miyazaki M, Ichinose N, Sugiura S, Kassai Y, Kanazawa H and Machida Y: A novel MR angiography technique: SPEED acquisition using half-Fourier RARE. J Magn Reson Imaging 8: 505-507, 1998.

18. Yokoyama K, Nitatori $\mathrm{T}$, Inaoka $\mathrm{S}$, Takahara $\mathrm{T}$ and Hachiya $\mathrm{J}$ : Non-contrast enhanced MR venography using 3D fresh blood imaging (FBI): initial experience. Radiat Med 19: 247-253, 2001.

19. Hudson TM, Enneking WF and Hawkins IF Jr: The value of angiography in planning surgical treatment of bone tumors. Radiology 138: 283-292, 1981 .

20. Swan JS, Weber DM, Korosec FR, Grist TM and Heiner JP: Combined MRI and MRA for limb salvage planning. J Comput Assist Tomogr 17: 339-342, 1993.

21. Lang P, Grampp S, Vahlensieck M, Johnston JO, Honda G, Rosenau W, Matthay KK, Peterfy C, Higgins CB, Genant HK, et al: Primary bone tumors: value of MR angiography for preoperative planning and monitoring response to chemotherapy. Am J Roentgenol 165: 135-142, 1995.

22. Ono A, Takada S, Kawai A, Kawakami Y, Taniguchi T, Shibuya O, Sano M and Miyazaki M: MR arteriography for pelvic to thigh region by swap phase encoding method. Japan J Magn Reson Med 22: 81-90, 2002 (in Japanese). 